\title{
Disentangling the effect of insemination and ovary development on the cuticular hydrocarbon profile in the bumblebee Bombus terrestris (Hymenoptera: Apidae)
}

\author{
Jan JANSEN ${ }^{1,2,3}$, Tamara PoKoRnY ${ }^{1,4}$, Thomas SCHMITT $^{1,5}$ \\ ${ }^{1}$ Department of Evolutionary Biology and Animal Ecology, Faculty of Biology, University of Freiburg, Hauptstr. 1, \\ 79104, Freiburg, Germany \\ ${ }^{2}$ Institute for Marine and Antarctic Studies, University of Tasmania, 20 Castray Esplanade, Battery Point 7004, Australia \\ ${ }^{3}$ Australian Antarctic Division, Department of the Environment, 203 Channel Hwy, Kingston 7050, Australia \\ ${ }^{4}$ Department of Evolutionary Ecology and Animal Biodiversity, Faculty of Biology and Biotechnology, University of \\ Bochum, Universitätsstr. 150, 44780, Bochum, Germany \\ ${ }^{5}$ Department of Animal Ecology and Tropical Biology, Biocenter, University of Würzburg, Am Hubland, 97074, \\ Würzburg, Germany
}

Received 30 September 2014 - Accepted 30 June 2015

\begin{abstract}
In many social insects, fertility of the reproductive caste is communicated via cuticular chemistry. Changes in cuticular profiles have been correlated to ovarian development. However, an effect of insemination has been neglected in the majority of studies so far. In Bombus terrestris, we stimulated virgin queens to found colonies without being inseminated and compared their cuticular hydrocarbon profile (CHC) to other females in different insemination and breeding statuses. The analysis of the CHC profiles showed an effect of both insemination and ovarian development on the cuticular profile. Thus, insemination besides ovarian development might be received by workers in bumblebees as a potential cue for fertility.
\end{abstract}

breeding status / cuticular profile / fertility / mating / virgin queen

\section{INTRODUCTION}

A multifunctional hydrocarbon-based layer is located on the cuticle of all so far investigated insects. While it is thought to have originated as a barrier against desiccation, cuticle abrasion, and microbial infection (Lockey 1988; St. Leger 1995; Gibbs 2002), in many insect taxa, such as Hymenoptera, it has additionally evolved to play a crucial role in chemical communication (Blomquist and Bagnères 2010). Cuticular hydrocarbons can serve as kairomones, as pheromones and as recognition cues for a variety of tasks

Corresponding author: J. Jansen, jan.jansen@utas.edu.au

Manuscript editor: Klaus Hartfelder
(Blomquist and Bagnères 2010). In eusocial Hymenoptera, information on intraspecific and interspecific membership, social hierarchy, and reproductive division of labor are encrypted in the cuticular profile (Howard and Blomquist 1982, 2005; Blomquist and Bagnères 2010). Thus, a wide range of factors has been found to affect the hydrocarbon profile: external factors such as food, humidity and temperature; social factors like colony membership; and physiological factors including age, ovarian development, and insemination (Bagnères and Wicker-Thomas 2010; van Zweden and d'Ettorre 2010; Liebig 2010).

The use of cuticular hydrocarbons (CHC) to signal females' reproductive status is widespread within eusocial Hymenoptera. Strong effects of fertility on the $\mathrm{CHC}$ profile and thus distinct queen specific $\mathrm{CHC}$ profiles have already been 
found in social wasps (e.g., Sledge et al. 2001, 2004), ants (e.g., Monnin et al. 1998; Peeters et al. 1999; Liebig et al. 2000; Heinze et al. 2002; Dietemann et al. 2003, 2005; Smith et al 2008, 2009), and bumblebees (Ayasse et al. 1995; Sramkova et al. 2008). However, in the majority of those studies, potential effects of insemination and ovarian development on $\mathrm{CHC}$ profiles were not investigated separately. A major problem is the inseparable connection between caste membership, ovarian development, and insemination, as usually only inseminated queens develop their ovaries and workers with fully developed ovaries are not inseminated. To be able to discriminate between the effects of insemination and ovary development, a model species needs to provide similar individuals in an inseminated and a noninseminated condition which can have fully developed or undeveloped ovaries. So far, only some primitively eusocial polygynous queenless ant species from the subfamily Ponerinae have allowed for such investigations (e.g., Lommelen et al. 2006; Cuvillier-Hot et al. 2002; Liebig et al. 2000). They lack physical castes and sterile workers are able to mate, lay eggs, and become sterile again. Findings in these studies suggest that the hydrocarbon profile is affected by ovarian activity but not by mating status, as workers that have recently stopped laying eggs have the same hydrocarbon profile as non-egg-laying workers and the chemical profile of gamergates and unmated workers with active ovaries is essentially the same (Lommelen et al. 2006). However, we still lack studies in other eusocial insects with physical castes like in bumblebees.

Colonies of the bumblebee species Bombus terrestris consist of one dominant queen that founds the colony after emerging from hibernation in spring. Only fertilized queens survive hibernation (Ayasse and Jarau 2014). The queen produces a large amount of sterile female workers and after some time, the colony reaches a switch point at which the queen starts to produce males and young queens. Later, some workers might also start to lay eggs, but as they are not inseminated, only haploid males hatch from these unfertilized eggs (Röseler and van Honk 1990). The oviposition rate of the queen increases with increasing colony size (Duchateau and Velthuis
1988), and the ovary development is linked to the breeding status (e.g., Bloch et al. 2000; Geva et al. 2005). Foraging workers, virgin, and nonbreeding queens have undeveloped ovaries, whereas breeding queens and dominant egglaying workers have fully developed ovaries (Ayasse et al. 1995; Bloch et al. 2000; Geva et al. 2005). Cuticular profiles of the different castes within one colony revealed an honest signaling of dominance and fertility: the more the ovaries of a worker are developed, the more their cuticular profile resembles the cuticular profile of the queen (Sramkova et al. 2008). Sramkova et al. (2008) were also able to show large differences in the cuticular profile depending on the breeding status of the queen, where nonbreeding queens differed from breeding queens in variety and amount of substances found on the cuticle.

In spite of recent progress in the understanding and composition of fertility cues there is still a need to disentangle the effect of ovary development and insemination that might affect a $\mathrm{CHC}$ profile. It might be incomplete if we base our research solely on comparing the $\mathrm{CHC}$ profiles of different castes. Therefore, we use an approach in which we induce egg laying in virgin queens of B. terrestris to gain $\mathrm{CHC}$ extracts from noninseminated colony-founding queens with fully developed ovaries. Finally, we compare the CHC profile of foraging bumblebee workers and four different groups of bumblebee queens to discriminate between potential effects of insemination and ovary development.

\section{MATERIAL AND METHODS}

\subsection{Insect collection and rearing}

For the comparative analysis of the cuticular hydrocarbon profiles, specimens were chosen from five different groups of female bumblebees: foraging workers, mated breeding queens, mated nonbreeding queens, virgin nonbreeding queens, and virgin breeding queens.

In early spring, nest-searching, nonbreeding queens and in summer, foraging workers of $B$. terrestris were caught in the botanical garden of the University of Freiburg, Germany. Twelve 
mated nest-searching, nonbreeding queens and twelve workers were freeze-killed immediately after their capture and kept at $-20{ }^{\circ} \mathrm{C}$ until extraction.

For rearing of bumblebee colonies, 176 mated nest-searching queens were placed into wooden rearing cages $(7 \times 12.5 \times 5 \mathrm{~cm})$ with a removable glass front and wired mesh bottom. The cages contained pollen and nectar substitute (APIInvert, Südzucker AG Mannheim/Ochsenfurt, Germany) provided ad libitum as well as a paste made out of powdered pollen and nectar substitute as base for laying eggs. They were kept in a climatized room under red light at $22{ }^{\circ} \mathrm{C}$ and $70 \%$ humidity. As soon as a queen had reared at least two workers, the colony was moved into a bigger wooden cage $(10 \times 26.5 \times 14 \mathrm{~cm})$ containing two equally sized compartments, one serving as a nest chamber, the other as a foraging area provisioned with nectar substitute and pollen ad libitum. Several colonies were kept until they were grown and produced males and virgin queens. Eight queens were freeze-killed when their colonies consisted of one to eight workers. Eight randomly selected virgin queens reared from three different colonies were also freeze-killed before they were 1 week old. Forty-two virgin queens were anesthetized for 30 min under $\mathrm{CO}_{2}$ atm. and thereafter started breeding without being inseminated. Eight of these virgin breeding queens were freeze-killed when their colonies consisted of two to five males. All freeze-killed specimens were kept at $-20{ }^{\circ} \mathrm{C}$ until extraction.

We estimated the ovary development of queens depending on their breeding status (Bloch et al. 2000; Geva et al. 2005) into two categories: undeveloped ovaries for nonbreeding queens and developed ovaries for breeding queens. All nonbreeding queens had neither raised workers nor had they laid eggs. All breeding queens were at the start of colony foundation with one to eight workers (virgin breeding queens produce males instead of workers). Colony sizes had to be kept small as we found that colonies of virgin queens collapse due to the lack of support given by males.

\subsection{Chemical analysis of cuticular hydrocarbons}

For $\mathrm{CHC}$ profile extraction, single specimens were transferred into 4-mL glass vials and extracted with pentane for $10 \mathrm{~min}$. The solvent was evaporated to approximately $1 \mathrm{~mL}$ using a gentle stream of nitrogen. The nonpolar hydrocarbons of each extract were separated from polar compounds using $3 \mathrm{~mL}$ SiOH-columns (Chromabond, Machery \&Nagel GmbH \& Co. $\mathrm{KG}$, Düren, Germany). Columns were conditioned with pentane before adding the extract. The hydrocarbons were eluted with three column equivalents of pentane.

For gas chromatography-mass spectrometry analysis, we used a Hewlett Packard HP 6890 Series GC System coupled to a Hewlett Packard HP 5973 Mass Selective Detector (Hewlett Packard, Waldbronn, Germany). The GC was equipped with a J \& W DB-1 fused silica capillary column $(30 \mathrm{~m} \times 9 \times 0.25 \mathrm{~mm}$ ID; $\mathrm{df}=0.25 \mu \mathrm{m} ; \mathrm{J} \&$ $\mathrm{W}$, Folsom, CA, USA). Temperature was programmed from 60 to $300{ }^{\circ} \mathrm{C}$ with $5{ }^{\circ} \mathrm{C} / \mathrm{min}$ heating rate and held for $10 \mathrm{~min}$ at $300{ }^{\circ} \mathrm{C}$. Helium was used as a carrier gas with a constant flow of $1 \mathrm{~mL} / \mathrm{min}$. Injection was carried out at $250{ }^{\circ} \mathrm{C}$ in the splitless mode for $1 \mathrm{~min}$. The electron impact mass spectra (EI-MS) were recorded at $70 \mathrm{eV}$ and $230{ }^{\circ} \mathrm{C}$. We used the Windows version of the ChemStation software package (Agilent Technologies, Böblingen, Germany) for data acquisition and processing.

Hydrocarbons were identified using a commercially available MS library (Wiley275, John Wiley and Sons Inc., NY, USA) and their diagnostic ions. For the identification of the double-bond positions in alkenes, we performed a dimethyl disulfide derivatization in accordance to Dunkelblum et al. (1985).

\subsection{Statistical analysis}

Prior to analysis, compounds which accounted for less than $0.01 \%$ of the relative peak area in all samples were discarded. If a compound accounted for more than $0.01 \%$ in one sample, this 
compound was included in the analysis although it may have accounted for less than $0.01 \%$ in other samples. Substances not occurring in at least $50 \%$ of all individuals of one group were removed from the dataset. Furthermore, we discarded compounds shorter than $\mathrm{C} 19$ as potential contaminants from the analysis in congruence with a former study (Sramkova et al. 2008).

Since relative peak areas of a sample are not statistically independent, we transformed the data according to Aitchison (1986). However, the original transformation procedure makes it necessary to exclude compounds that do not occur in all samples. Thus, peaks that are zero in some samples but are present in other samples would not have been considered. Therefore, we modified the transformation to avoid undefined values for peaks with an area of zero:

$\log 10\left(\frac{\text { relative peak area }}{\text { geometric mean relative peak area }}+1\right)$

We reduced the number of variables for the linear discriminant analysis using principal component analysis because of the large number of peaks relative to the sample size. Only principal components with an eigenvalue $>1$ were used to perform the discriminant analysis. The resulting variables were subsequently analyzed in a leave-one-out cross-validation to test whether individuals can be identified correctly into their group according to their $\mathrm{CHC}$ profile.

To determine which substances are most important for the separation of the groups, we analyzed the relative composition of the $\mathrm{CHC}$ profiles using a random forest classification (Breiman 2001) (number of trees $=500$ ). Five random forests were constructed: first, we compared all five groups; second, we compared virgin queens with mated queens; third, we compared breeding queens with nonbreeding queens; fourth and fifth, we compared virgin nonbreeding queens to mated nonbreeding queens and virgin breeding queens, respectively. We used the mean decrease of accuracy to interpret substance importance as suggested by Cutler et al. (2007). The mean decrease in accuracy for a variable is the normalized difference of the classification accuracy for the out-of-bag data when the data for that variable is included as observed, and the classification accuracy for the out-of-bag data when the values of the variable in the out-of-bag data have been randomly permuted.

Data were analyzed using $\mathrm{R}$ version 3.1.2 ( $\mathrm{R}$ Core Team 2014) with the packages "Hmisc" (Harrell 2011), "MASS" (Venables and Ripley 2002), "randomForest" (Liaw and Wiener 2002), and "vegan" (Oksanen et al. 2011).

\section{RESULTS}

We characterized the hydrocarbons of 69 peaks extracted from the cuticle of the investigated groups of $B$. terrestris females (Table I). Principal component analysis (PCA) was conducted using the transformed relative amount of each of these peaks and resulted in 12 principal components (PCs) having an eigenvalue $>1$ and explaining $88.49 \%$ of variance. Linear discriminant analysis (DA) calculated four discriminant functions (DF) from these $12 \mathrm{PCs}$ and resulted in a complete separation of all five groups (Wilk's $\Lambda=0.00004 ; \mathrm{df}=48 ; F=35.06 ; P<0.001$; Figure 1). DF 1 represents $48.53 \%$ of variance and is sufficient to separate all groups of female bumblebees (Table II). DF 2 represents a further $27.56 \%$ of variance and separates all groups with the exception of workers and virgin nonbreeding queens. In leave-one-out cross-validation, all individuals were classified correctly into their groups.

Random forest (RF) calculated an out-of-bag (OOB) estimate of error rate at $2.04 \%$ (1 out of 49 individuals misclassified) for the classification of five groups (Table III). One virgin nonbreeding queen was incorrectly classified into the group of workers. The substances with the highest importance are 9-, 7-C29en; 5-C27en; n-C22; a C33dien with unknown double-bond positions and a C30dien with unknown double-bond positions (Figure 2). The second RF classification with queens grouped according to whether they are virgin or mated had an $\mathrm{OOB}$ estimate of error rate of $2.7 \%$ (1 out of 37 individuals 
Table I. Mean of relative peak areas (in \%) of hydrocarbons found on the cuticle of five different female bumblebee groups.

\begin{tabular}{|c|c|c|c|c|c|c|c|c|c|c|c|}
\hline \multirow{2}{*}{$\begin{array}{l}\text { Ret. } \\
\text { index }\end{array}$} & \multirow{2}{*}{$\begin{array}{l}\text { Substance } \\
\mathrm{n}-\mathrm{C} 19\end{array}$} & \multicolumn{2}{|c|}{ Worker } & \multicolumn{2}{|c|}{$\begin{array}{c}\text { Virgin } \\
\text { nonbreeding } \\
\text { queen }\end{array}$} & \multicolumn{2}{|c|}{$\begin{array}{l}\text { Virgin } \\
\text { breeding } \\
\text { queen }\end{array}$} & \multicolumn{2}{|c|}{$\begin{array}{l}\text { Mated } \\
\text { breeding } \\
\text { queen }\end{array}$} & \multicolumn{2}{|c|}{$\begin{array}{c}\text { Mated } \\
\text { nonbreeding } \\
\text { queen }\end{array}$} \\
\hline & & 0.03 & \pm 0.01 & 0.05 & \pm 0.02 & 0.02 & \pm 0.01 & 0.02 & \pm 0.02 & 0.13 & \pm 0.40 \\
\hline 2000 & $\mathrm{n}-\mathrm{C} 20$ & 0.09 & \pm 0.04 & 0.05 & \pm 0.02 & 0.03 & \pm 0.01 & 0.02 & \pm 0.01 & 0.04 & \pm 0.02 \\
\hline 100 & $\mathrm{n}-\mathrm{C} 21$ & 1.08 & \pm 0.49 & 1.28 & \pm 0.31 & 1.42 & \pm 0.56 & 1.57 & \pm 0.76 & 3.28 & \pm 2.07 \\
\hline 200 & $\mathrm{n}-\mathrm{C} 22$ & 0.24 & \pm 0.04 & 0.25 & \pm 0.05 & 0.32 & \pm 0.11 & & & 0.28 & \pm 0.11 \\
\hline 2271 & 9-C23en & 0.33 & \pm 0.21 & 0.35 & \pm 0.11 & 0.23 & \pm 0.14 & 0.31 & \pm 0.26 & 0.52 & \pm 0.47 \\
\hline 2278 & $\mathrm{a}-\mathrm{C} 23 \mathrm{en}$ & 0.03 & \pm 0.02 & 0.03 & \pm 0.01 & 0.02 & \pm 0.01 & 0.02 & \pm 0.02 & 0.02 & \pm 0.06 \\
\hline 292 & b-C23en & 0.02 & \pm 0.01 & 0.05 & \pm 0.01 & 0.04 & \pm 0.01 & 0.04 & \pm 0.01 & 0.04 & \pm 0.01 \\
\hline 2300 & $\mathrm{n}-\mathrm{C} 23$ & 11.20 & \pm 2.36 & 14.83 & \pm 2.49 & 15.76 & \pm 3.40 & 14.33 & \pm 4.44 & 12.11 & \pm 2.71 \\
\hline 2372 & $\begin{array}{c}3 \mathrm{~m}-\mathrm{C} 23 \\
\text { and a- } \\
\mathrm{C} 24 \mathrm{en}\end{array}$ & 0.08 & \pm 0.10 & 0.01 & & 001 & +001 & 0.02 & \pm 0.01 & 0.02 & \pm 0.04 \\
\hline 2400 & $\mathrm{n}-\mathrm{C} 24$ & 0.34 & \pm 0.07 & 0.40 & \pm 0.10 & 0.51 & \pm 0.23 & 0.28 & \pm 0.12 & 0.23 & \pm 0.06 \\
\hline 2450 & 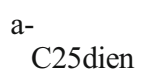 & $<0.01$ & \pm 0.01 & 0.02 & \pm 0.02 & $<0.01$ & $\pm<0.01$ & 0.01 & \pm 0.01 & 0.02 & \pm 0.02 \\
\hline 2462 & $4 \mathrm{~m}-\mathrm{C} 24$ & 0.16 & \pm 0.35 & $<0.01$ & $\pm<0.01$ & $<0.01$ & \pm 0.01 & & & & \\
\hline 467 & 11-C25en & 0.07 & \pm 0.10 & 0.35 & & 0.09 & & 0.17 & \pm 0.10 & 0.41 & \pm 0.25 \\
\hline 2472 & 9-C25en & 0.46 & \pm 0.24 & 0.38 & \pm 0.09 & 0.17 & \pm 0.08 & 0.37 & \pm 0.70 & 0.63 & \pm 1.47 \\
\hline 2479 & 7-C25en & 0.11 & \pm 0.05 & 0.11 & \pm 0.05 & 0.03 & \pm 0.01 & 0.09 & \pm 0.19 & 0.13 & \pm 0.19 \\
\hline 2492 & $\mathrm{a}-\mathrm{C} 25 \mathrm{en}$ & 0.07 & \pm 0.14 & 0.05 & \pm 0.01 & 0.03 & \pm 0.01 & 0.03 & \pm 0.03 & 0.03 & \pm 0.01 \\
\hline 2500 & $n-C 25$ & 11.82 & \pm 3.00 & 14.13 & & & & & & 8.70 & \pm 2.31 \\
\hline 2568 & $\mathrm{a}-\mathrm{C} 26 \mathrm{en}$ & 0.01 & \pm 0.01 & 0.07 & \pm 0.04 & 0.02 & \pm 0.01 & 0.04 & \pm 0.03 & 0.09 & \pm 0.06 \\
\hline 2574 & $\begin{array}{c}3 \mathrm{~m}-\mathrm{C} 25 \\
\text { and 9- } \\
\text { C26en }\end{array}$ & 0.22 & \pm 0 & 0.09 & \pm 0 & 0.03 & \pm 0.01 & 0.04 & \pm 0.02 & 0.06 & \pm 0.04 \\
\hline 2582 & b-C26en & & & 0.04 & \pm 0.02 & 0.01 & $\pm<0.01$ & 0.02 & \pm 0.02 & 0.02 & \pm 0.02 \\
\hline 2600 & $n-C 26$ & 0.98 & \pm 0.35 & 0.73 & \pm 0.16 & 0.89 & & 0.63 & \pm 0.14 & 0.58 & \pm 0.14 \\
\hline 2645 & $\begin{array}{l}a-; b- \\
\text { C27dien }\end{array}$ & 0.06 & \pm 0.11 & 0.34 & \pm 0.22 & $0 \Omega$ & \pm 0.03 & 0.09 & \pm 0.08 & 0.24 & \pm 0.17 \\
\hline 2652 & $\begin{array}{l}\mathrm{c}- \\
\mathrm{C} 27 \mathrm{dien}\end{array}$ & 0.03 & \pm 0.06 & 0.20 & \pm 0.14 & 0.02 & $=0.02$ & 0.04 & \pm 0.03 & 0.09 & \pm 0.06 \\
\hline 2659 & $\begin{array}{l}4 \mathrm{~m}-\mathrm{C} 26 \\
\text { and d- } \\
\mathrm{C} 27 \text { dien }\end{array}$ & 0.06 & \pm 0.13 & 0.15 & \pm 0.10 & 0.01 & \pm 0.01 & 0.02 & \pm 0.02 & 0.04 & \pm 0.04 \\
\hline 2666 & $\begin{array}{r}13-; 11- \\
\text { C27en }\end{array}$ & 0.52 & \pm 0.91 & 3.68 & \pm 1.80 & 1.42 & \pm 0.61 & 2.70 & \pm 1.85 & 4.96 & \pm 2.65 \\
\hline 2674 & 9-C27en & 7.28 & \pm 4.78 & 4.48 & \pm 1.30 & 1.20 & \pm 0.68 & 1.20 & \pm 0.77 & 1.79 & \pm 1.49 \\
\hline 2680 & 7-C27en & 0.70 & \pm 0.28 & 1.38 & \pm 0.52 & 0.31 & \pm 0.15 & 0.62 & \pm 0.90 & 0.63 & \pm 0.53 \\
\hline 2690 & 5-C27en & 0.38 & \pm 0.76 & 0.09 & \pm 0.08 & 0.07 & \pm 0.02 & 0.08 & \pm 0.05 & & \\
\hline 2700 & $\mathrm{n}-\mathrm{C} 27$ & 26.35 & \pm 10.1 & 19.08 & \pm 3.26 & 18.77 & \pm 2.58 & 19.97 & \pm 2.98 & 17.35 & \pm 5.02 \\
\hline 2744 & ${ }^{\mathrm{a}-} \mathrm{C} 28 \mathrm{dien}$ & 0.01 & \pm 0.02 & 0.02 & \pm 0.02 & 0.01 & \pm 0.01 & 0.02 & \pm 0.01 & 0.03 & \pm 0.02 \\
\hline 2749 & $\begin{array}{l}\text { b- } \\
\text { C28dien }\end{array}$ & 0.01 & \pm 0.03 & 0.02 & \pm 0.02 & 0.03 & \pm 0.01 & 0.03 & \pm 0.01 & 0.03 & \pm 0.02 \\
\hline 2766 & $\begin{array}{r}\text { 13-; 11- } \\
\text { C28en }\end{array}$ & 0.05 & \pm 0.09 & 0.16 & \pm 0.10 & 0.16 & \pm 0.04 & 0.22 & \pm 0.11 & 0.36 & \pm 0.18 \\
\hline
\end{tabular}


Table I (continued)

\begin{tabular}{|c|c|c|c|c|c|c|c|c|c|c|c|}
\hline \multirow{2}{*}{$\begin{array}{l}\text { Ret. } \\
\text { index } \\
2775\end{array}$} & \multirow{2}{*}{$\begin{array}{l}\text { Substance } \\
9-\mathrm{C} 28 \mathrm{en}\end{array}$} & \multicolumn{2}{|c|}{ Worker } & \multicolumn{2}{|c|}{$\begin{array}{c}\text { Virgin } \\
\text { nonbreeding } \\
\text { queen }\end{array}$} & \multicolumn{2}{|c|}{$\begin{array}{l}\text { Virgin } \\
\text { breeding } \\
\text { queen }\end{array}$} & \multicolumn{2}{|c|}{$\begin{array}{l}\text { Mated } \\
\text { breeding } \\
\text { queen }\end{array}$} & \multicolumn{2}{|c|}{$\begin{array}{c}\text { Mated } \\
\text { nonbreeding } \\
\text { queen }\end{array}$} \\
\hline & & 0.69 & \pm 0.32 & 0.42 & \pm 0.10 & 0.30 & \pm 0.09 & 0.27 & \pm 0.09 & 0.31 & \pm 0.07 \\
\hline 2783 & $\mathrm{a}-\mathrm{C} 28 \mathrm{en}$ & 0.01 & \pm 0.02 & 0.01 & \pm 0.02 & & & 0.03 & \pm 0.04 & 0.05 & \pm 0.02 \\
\hline 2800 & $\mathrm{n}-\mathrm{C} 28$ & 0.61 & \pm 0.26 & 0.45 & \pm 0.09 & 0.67 & \pm 0.12 & 0.57 & \pm 0.16 & 0.66 & \pm 0.23 \\
\hline 2844 & $\begin{array}{l}\text { a-; b- } \\
\text { C29dien }\end{array}$ & 0.72 & \pm 1.38 & 1.12 & \pm 0.56 & 1.21 & \pm 0.47 & 1.27 & \pm 0.55 & 1.60 & \pm 0.88 \\
\hline 2856 & $\begin{array}{l}\text { c- } \\
\text { C29dien }\end{array}$ & 0.27 & \pm 0.50 & 0.47 & \pm 0.23 & 0.37 & \pm 0.18 & 0.29 & \pm 0.18 & 0.32 & \pm 0.21 \\
\hline 2867 & $\begin{array}{l}\text { 13-; 11- } \\
\text { C29en }\end{array}$ & 1.91 & \pm 3.06 & 4.03 & \pm 1.51 & 5.66 & \pm 1.68 & 6.52 & \pm 3.16 & 8.47 & \pm 5.86 \\
\hline 2872 & $\begin{array}{l}\text { 9-; 7- } \\
\text { C29en }\end{array}$ & 20.04 & \pm 7.23 & 13.89 & \pm 2.36 & 10.76 & \pm 2.65 & 9.81 & \pm 3.40 & 9.83 & \pm 4.72 \\
\hline 2886 & 5-C29en & 0.81 & \pm 2.41 & & & & & 0.10 & \pm 0.02 & & \\
\hline 2900 & $\mathrm{n}-\mathrm{C} 29$ & 6.74 & \pm 3.17 & 7.50 & \pm 1.54 & 10.96 & \pm 3.20 & 12.30 & \pm 3.45 & 13.40 & \pm 5.26 \\
\hline 2944 & ${ }^{\mathrm{a}-}$ C30dien & 0.03 & \pm 0.06 & 0.02 & \pm 0.03 & 0.08 & \pm 0.02 & 0.07 & \pm 0.04 & 0.09 & \pm 0.04 \\
\hline 2952 & $\begin{array}{l}\mathrm{b}- \\
\mathrm{C} 30 \text { dien }\end{array}$ & 0.02 & \pm 0.04 & 0.01 & \pm 0.02 & 0.05 & \pm 0.01 & 0.04 & \pm 0.02 & 0.03 & \pm 0.03 \\
\hline 2960 & C- & & & & & 0.02 & $\pm<0.01$ & 0.01 & \pm 0.01 & $<0.01$ & $\pm<0.01$ \\
\hline 2966 & a-C30en & & & & & 0.07 & \pm 0.04 & 0.05 & \pm 0.06 & & \\
\hline 2971 & 9-C30en & 0.09 & \pm 0.14 & 0.14 & \pm 0.07 & 0.17 & \pm 0.03 & 0.24 & \pm 0.09 & 0.39 & \pm 0.22 \\
\hline 2977 & 7-C30en & 0.13 & \pm 0.09 & 0.18 & \pm 0.05 & 0.23 & \pm 0.07 & 0.14 & \pm 0.10 & 0.10 & \pm 0.10 \\
\hline 3000 & $\mathrm{n}-\mathrm{C} 30$ & 0.05 & \pm 0.04 & 0.06 & \pm 0.02 & 0.20 & \pm 0.04 & 0.15 & \pm 0.10 & 0.17 & \pm 0.19 \\
\hline 3043 & ${ }^{\mathrm{a}-}$ C31dien & 0.31 & \pm 0.52 & 0.14 & \pm 0.16 & 0.83 & \pm 0.48 & 0.91 & \pm 0.63 & 0.84 & \pm 0.66 \\
\hline 3048 & $\begin{array}{l}\text { b- } \\
\text { C31dien }\end{array}$ & 0.22 & \pm 0.47 & 0.36 & \pm 0.15 & 0.64 & \pm 0.32 & 0.38 & \pm 0.46 & 0.21 & \pm 0.36 \\
\hline 3053 & c- & 0.54 & \pm 0.95 & 0.52 & \pm 0.13 & 0.99 & \pm 0.42 & 0.77 & \pm 0.60 & 0.61 & \pm 0.46 \\
\hline 3060 & $\begin{array}{l}\mathrm{d}- \\
\mathrm{C} 31 \text { dien }\end{array}$ & 0.34 & \pm 0.57 & 0.46 & \pm 0.11 & 0.74 & \pm 0.28 & 0.58 & \pm 0.55 & 0.29 & \pm 0.35 \\
\hline 3072 & 9-C31en & 1.61 & \pm 2.44 & 2.79 & \pm 0.82 & 4.44 & \pm 1.25 & 4.67 & \pm 2.02 & 6.04 & \pm 3.27 \\
\hline 3080 & 7-C31en & 1.61 & \pm 1.56 & 3.09 & \pm 0.67 & 3.83 & \pm 1.41 & 2.28 & \pm 1.86 & 1.26 & \pm 1.35 \\
\hline 3100 & n-C31 & 0.33 & \pm 0.33 & 0.42 & \pm 0.20 & 1.48 & \pm 0.61 & 1.54 & \pm 1.32 & 1.78 & \pm 2.00 \\
\hline 3146 & unknown & & & $<0.01$ & \pm 0.01 & 0.01 & \pm 0.02 & 0.02 & \pm 0.02 & & \\
\hline 3153 & ${ }^{\mathrm{a}-}$ C32dien & $<0.01$ & \pm 0.01 & 0.01 & \pm 0.02 & 0.05 & \pm 0.03 & 0.04 & \pm 0.03 & 0.01 & \pm 0.02 \\
\hline 3167 & $\begin{array}{l}\text { b- } \\
\text { C32dien }\end{array}$ & & & & & 0.05 & \pm 0.04 & 0.05 & \pm 0.07 & 0.01 & \pm 0.02 \\
\hline 3171 & a-C32en & & & 0.04 & \pm 0.05 & 0.02 & \pm 0.02 & 0.03 & \pm 0.03 & 0.04 & \pm 0.03 \\
\hline 3177 & b-C32en & 0.01 & \pm 0.02 & & & 0.03 & \pm 0.04 & $<0.01$ & \pm 0.01 & $<0.01$ & \pm 0.01 \\
\hline 3200 & $n-C 32$ & & & & & 0.03 & \pm 0.02 & 0.01 & \pm 0.01 & 0.01 & \pm 0.02 \\
\hline 3238 & ${ }^{\mathrm{a}-}$ C33dien & $<0.01$ & \pm 0.01 & & & 0.04 & \pm 0.11 & 0.14 & \pm 0.22 & 0.04 & \pm 0.08 \\
\hline 3244 & b- & 0.06 & \pm 0.10 & 0.04 & \pm 0.07 & 0.32 & \pm 0.16 & 0.31 & \pm 0.29 & 0.11 & \pm 0.17 \\
\hline 3250 & $\begin{array}{l}\mathrm{c}- \\
\mathrm{C} 33 \text { dien }\end{array}$ & & & 0.24 & \pm 0.14 & & & & & $<0.01$ & $\pm<0.01$ \\
\hline
\end{tabular}


Table I (continued)

\begin{tabular}{cccccccccccc}
\hline $\begin{array}{c}\text { Ret. } \\
\text { index }\end{array}$ & Substance & Worker & \multicolumn{2}{c}{$\begin{array}{c}\text { Virgin } \\
\text { nonbreeding } \\
\text { queen }\end{array}$} & $\begin{array}{c}\text { Virgin } \\
\text { breeding } \\
\text { queen }\end{array}$ & $\begin{array}{c}\text { Mated } \\
\text { breeding } \\
\text { queen }\end{array}$ & $\begin{array}{c}\text { Mated } \\
\text { nonbreeding } \\
\text { queen }\end{array}$ \\
\hline 3256 & $\begin{array}{c}\text { d- } \\
\text { C33dien }\end{array}$ & 0.04 & \pm 0.07 & 0.19 & \pm 0.10 & 0.21 & \pm 0.10 & 0.24 & \pm 0.25 & 0.09 & \pm 0.14 \\
3268 & $\begin{array}{c}13-; 11-; \\
\text { 9- }\end{array}$ & 0.04 & \pm 0.08 & 0.38 & \pm 0.25 & 0.31 & \pm 0.25 & 0.35 & \pm 0.25 & 0.26 & \pm 0.32 \\
& $\begin{array}{l}\text { C33en } \\
3279\end{array}$ & & & & & & & & & & \\
3300 & 7-C33en & & & 0.16 & \pm 0.08 & 0.52 & \pm 0.27 & 0.23 & \pm 0.23 & 0.08 & \pm 0.12 \\
& n-C33 & & & & & 0.06 & \pm 0.03 & 0.04 & \pm 0.07 & 0.03 & \pm 0.09 \\
\hline
\end{tabular}

IUPAC abbreviations are used for the substances. Kovats retention indices (Ret. index) are given for each substance. The letters a,b,c,d are unidentified double-bond positions in alkenes and alkadienes

misclassified). A C23en and a C28en both with an unknown double-bond position and 7-C30en were the most important substances in the classification of queens of different mating status (Figure 3a). For the third comparison, where queens are grouped according to whether they are breeding or not, RF calculated an OOB estimate of error rate of $0 \%$ (all individuals were correctly classified) and the most important substances to separate these groups are a C30dien with unknown double-bond positions, 5-C27en, two different C28dien with unknown double-

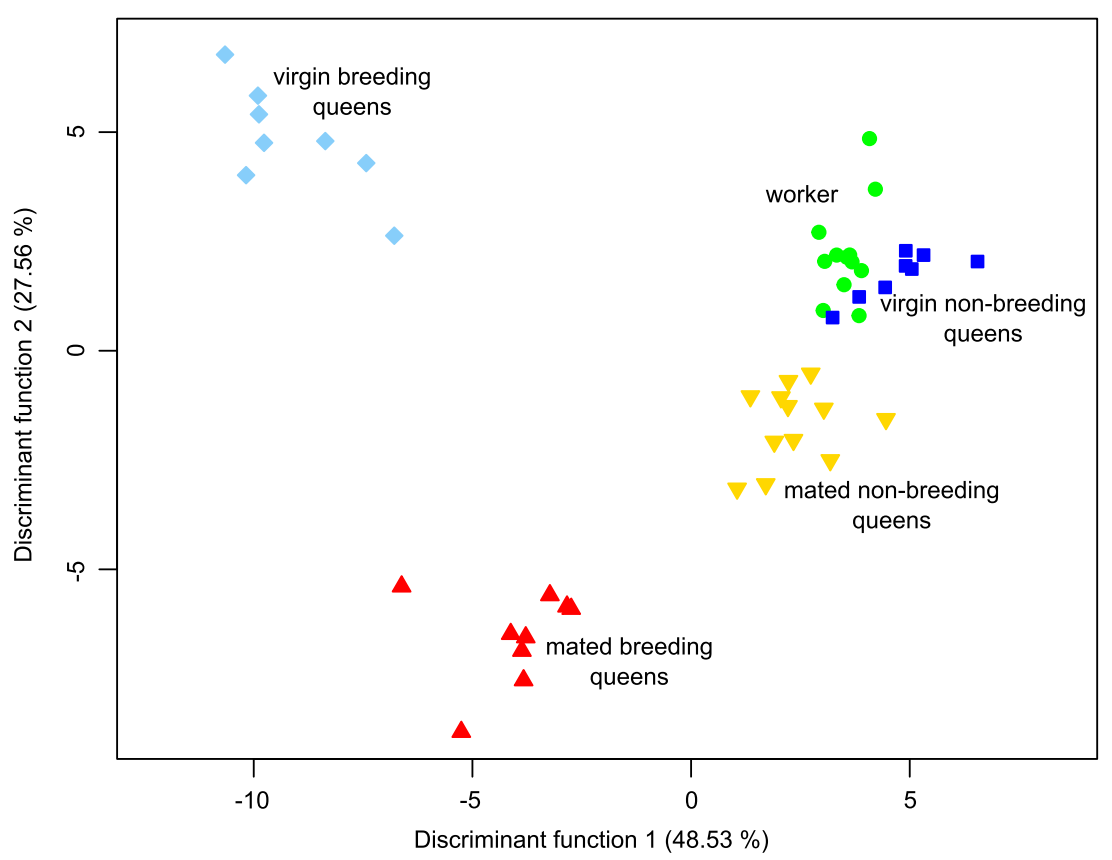

Figure 1. Linear discriminant analysis of the cuticular hydrocarbon profiles of five groups of female bumblebees: workers, virgin nonbreeding queens, virgin breeding queens, mated breeding queens, and mated nonbreeding queens. The first two discriminant functions are shown for the five groups. The analysis is based on 12 principal components with an eigenvalue $>1$. 
Table II. Linear discriminant analysis of the cuticular hydrocarbons profiles of five groups of female bumblebees: workers, virgin nonbreeding queens, virgin breeding queens, mated breeding queens, and mated nonbreeding queens. Comparison of groups and whether they can be separated by single discriminant functions (DF).

\begin{tabular}{llllll}
\hline \multicolumn{1}{c}{ Group } & \multicolumn{1}{c}{ Group } & DF1 & DF2 & DF3 & DF4 \\
\hline Worker & Virgin nonbreeding queen & $* *$ & n.s. & n.s. & $* * *$ \\
Worker & Virgin breeding queen & $* * *$ & $* *$ & $* * *$ & $* * *$ \\
Worker & Mated breeding queen & $* * *$ & $* * *$ & n.s. & $* * *$ \\
Worker & Mated nonbreeding queen & $* *$ & $* * *$ & $* * *$ & $* * *$ \\
Virgin nonbreeding queen & Virgin breeding queen & $* * *$ & $* * *$ & $* * *$ & $* * *$ \\
Virgin nonbreeding queen & Mated breeding queen & $* * *$ & $* * *$ & $*$ & $* * *$ \\
Virgin nonbreeding queen & Mated nonbreeding queen & $* * *$ & $* * *$ & $* * *$ & $* * *$ \\
Virgin breeding queen & Mated breeding queen & $* * *$ & $* * *$ & $* * *$ & n.s. \\
Virgin breeding queen & Mated nonbreeding queen & $* * *$ & $* * *$ & $* * *$ & n.s. \\
Mated breeding queen & Mated nonbreeding queen & $* * *$ & $* * *$ & $* * *$ & n.s. \\
\hline
\end{tabular}

DF were tested with a Wilcoxon signed-rank test and the $P$ values adjusted with Holm-Bonferroni n.s. not significant; $* P<0.05 ; * * P<0.01 ; * * * P<0.001$

bond positions, and n-C33 (Figure 3b). Classifying the substance importance in the separation of virgin nonbreeding queens and mated nonbreeding queens (Figure 4a) reveals an important influence of 7-C30en, a C33dien with unknown double-bond positions, C29en with double-bonds at positions seven and nine as well as a $\mathrm{C} 23$ en with an unknown double bond position. The OOB estimate error rate is $0 \%$. In the comparison between virgin nonbreeding queens and virgin breeding queens (Figure 4b), C33dien and C30dien, both with unknown double- bond positions, 5-C27en, and n-C33 are most influential. No individuals were misclassified.

\section{DISCUSSION}

The comparative analysis of the cuticular hydrocarbon (CHC) profiles using a linear discriminant analysis (DA) reveals a clear separation of all tested groups of Bombus terrestris females. As these five groups consist of a group of foraging workers as well as four groups of queens with different breeding statuses and with different statuses

Table III. Confusion matrix of classification errors from a random forest with all groups of female bumblebees.

\begin{tabular}{|c|c|c|c|c|c|c|}
\hline $\begin{array}{l}\text { Original } \\
\text { Predicted }\end{array}$ & Worker & $\begin{array}{c}\text { Virgin } \\
\text { non-breeding } \\
\text { queen }\end{array}$ & $\begin{array}{l}\text { Virgin } \\
\text { breeding } \\
\text { queen }\end{array}$ & $\begin{array}{l}\text { Mated } \\
\text { breeding } \\
\text { queen }\end{array}$ & $\begin{array}{c}\text { Mated } \\
\text { non-breeding } \\
\text { queen }\end{array}$ & $\begin{array}{c}\text { Classification } \\
\text { error }\end{array}$ \\
\hline Worker & 12 & 0 & 0 & 0 & 0 & 0 \\
\hline V. non-breeding queen & 1 & 7 & 0 & 0 & 0 & 0.125 \\
\hline V. breeding queen & 0 & 0 & 8 & 0 & 0 & 0 \\
\hline M. breeding queen & 0 & 0 & 0 & 9 & 0 & 0 \\
\hline M. non-breeding queen & 0 & 0 & 0 & 0 & 12 & 0 \\
\hline
\end{tabular}


Substance importance: separation of all groups

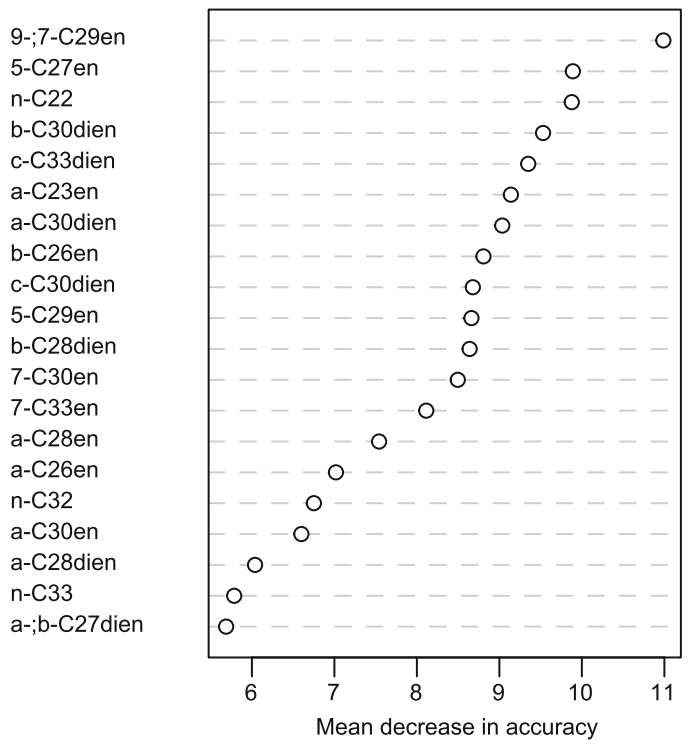

Figure 2. Variable importance plot from a random forest classification of cuticular hydrocarbons used in the identification of group membership of five groups of female bumblebees: workers, virgin nonbreeding queens, virgin breeding queens, mated breeding queens, and mated nonbreeding queens. Higher values of mean decrease in accuracy indicate higher importance of the variable for classification.

of insemination, we have evidence that the setup used in our experiments allows to disentangle the effects of ovarian development and insemination on $\mathrm{CHC}$ profiles.

The most pronounced differences among the different groups of $B$. terrestris queens appeared between virgin breeding queens, mated breeding queens, and all other groups. $48.53 \%$ of the variance of the DA explains almost exclusively the difference between breeding females and nonbreeding females. Hence, a strong effect of ovary development on the $\mathrm{CHC}$ profile can be concluded from these differences. Alkadienes are the most common group of substances that contribute to the separation between breeding and nonbreeding queens with n-C33 and 5-C27en also playing an important role. This result is supported by the fact that several alkadienes contribute most to the separation of virgin nonbreeding queens and virgin breeding queens. So far, studies in bumblebees have found a large amount of different substance classes such as alkanes, alkenes, aldehydes, methyl-branched alkanes, and wax-type esters to allow separation between groups (Ayasse et al. 1995; Sramkova et al. 2008). Furthermore, in different eusocial insects different substances seem to be driving separation between groups (e.g., Sledge et al. 2001; Smith et al. 2015). However, in many of the studies of eusocial insects where an influence of ovary development had been found, it was not investigated independently from possible effects like caste membership or insemination. Our results suggest that the differences in the $\mathrm{CHC}$ profiles between queens with the same status of insemination are most likely caused solely by the difference in their ovary development.

While ovary development has a strong effect on the CHC profile, our results also reveal an effect of insemination. The DA shows that mated queens and virgin queens are clearly separated from each other, mainly expressed on DF 2 which accounts for $27.56 \%$ of variance. Furthermore, the effect of insemination seems to be larger between breeding queens than between nonbreeding queens which might hint toward an interaction between the two effects. Interestingly, with a few exceptions, alkenes with a double-bond position on C7 (identified using DMDS derivatizations and/or retention indices) play a major role in separating inseminated from non-inseminated queens. 7alkenes are also responsible for the separation of virgin nonbreeding queens and mated nonbreeding queens. This might hint toward an effect of insemination on the regulation of C7 specific desaturases.

Insemination has been shown to affect the chemical profile in only a few other species. In Drosophila melanogaster, mating alters the $\mathrm{CHC}$ profiles of females (Everaerts et al. 2010). In contrast, studies in queenless ponerine ants, where individuals of all different statuses of fertility can occur naturally, did not find any effect of mating 
a

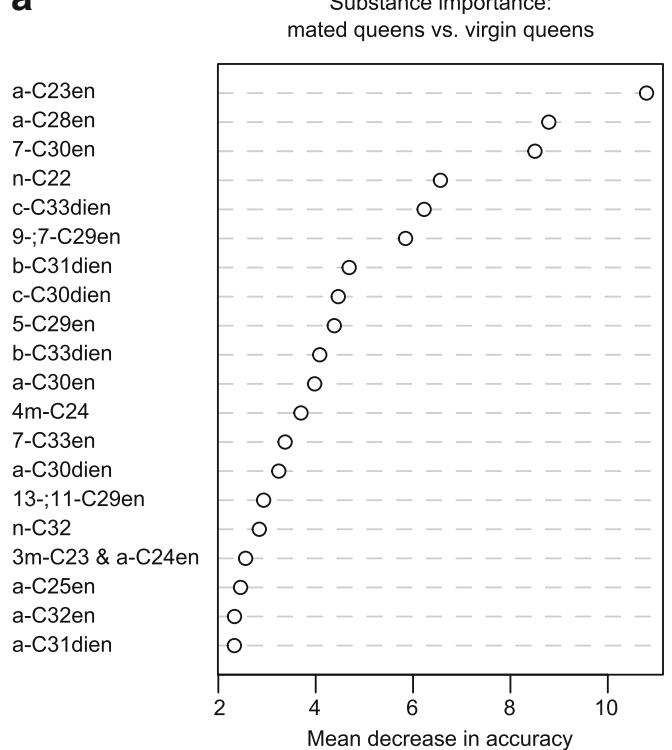

b

b-C30dien
5-C27en
b-C28dien
a-C28dien
$n-C 33$
a-C32dien
$n-C 22$
b-C32dien
9-;7-C29en
a-C25dien
c-C30dien
a-C30en
5-C29en
n-C32
unknown
3m-C23 \& a-C24en
a-C30dien
9-C30en
a-C25en
a-C28en

Substance importance: breeding queens vs.non-breeding queens

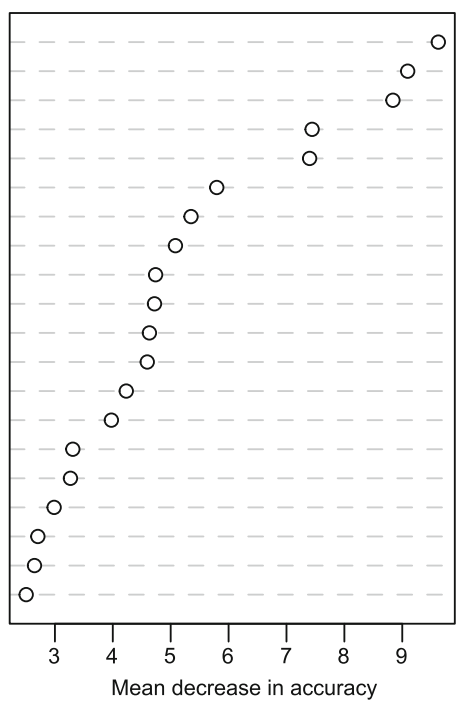

Figure 3. Variable importance plot from a random forest classification of cuticular hydrocarbons used in the identification of group membership of two groups of female bumblebees: a virgin queens (virgin breeding and virgin nonbreeding queens) versus mated queens (mated breeding and mated nonbreeding queens), $\mathbf{b}$ breeding queens (virgin and mated breeding queens) versus nonbreeding queens (virgin and mated nonbreeding queens). Higher values of mean decrease in accuracy indicate higher importance of the variable for classification.

on the CHC profile (e.g., Peeters et al. 1999; Liebig et al. 2000; Heinze et al. 2002). To our knowledge, there are so far no studies in Apidae which show an effect of insemination on the $\mathrm{CHC}$ profile. However, in the honeybee Apis mellifera, the chemical profile of Dufour's gland, which contains a specific number of polar substances, is affected by the number of matings (Richard et al. 2011). Thus, workers are more attracted to the extract of inseminated queens than to that of virgin queens, and more attracted to multiplemated queens than to single-mated queens. Richard et al. (2011) further found that differences in behavioral responses appeared to not be triggered by pronounced changes in individual chemicals but rather "by subtle changes in the overall chemical profile". While the Dufour's gland chemical profile is associated with the number of matings, the mandibular gland profile is associated with both the number of matings and the quality of the insemination (Niño et al. 2013). Effects of insemination on the cuticular profile have not yet been shown in $B$. terrestris; however, there are other effects on life history traits: insemination can have a life-shortening effect and it influences the hibernation survival rate (Baer and Schmid-Hempel 2005; Greeff and SchmidHempel 2008; Ayasse and Jarau 2014).

Besides ovary development and insemination, there might be also a possible effect of age and caste membership on the $\mathrm{CHC}$ profile. Age has been shown to strongly affect the $\mathrm{CHC}$ profile in insects mainly in the first week after emerging (e.g., Cuvillier-Hot et al. 2001; Mpuru et al. 2001; Panek et al. 2001). Later in life, differences in the $\mathrm{CHC}$ profile affected by age are often hard to measure as age comes along with other factors such as task, fertility, and dominance (Wilson 1963; Robinson 1992; Keller and Nonacs 1993). Thus, effects of age on the CHC profiles cannot be completely ruled out due to the setup of our experiments. The virgin nonbreeding queens in our experiment were less than 1 week old, and hence are likely to be of an age where drastic changes of their $\mathrm{CHC}$ profile can occur. However, the 


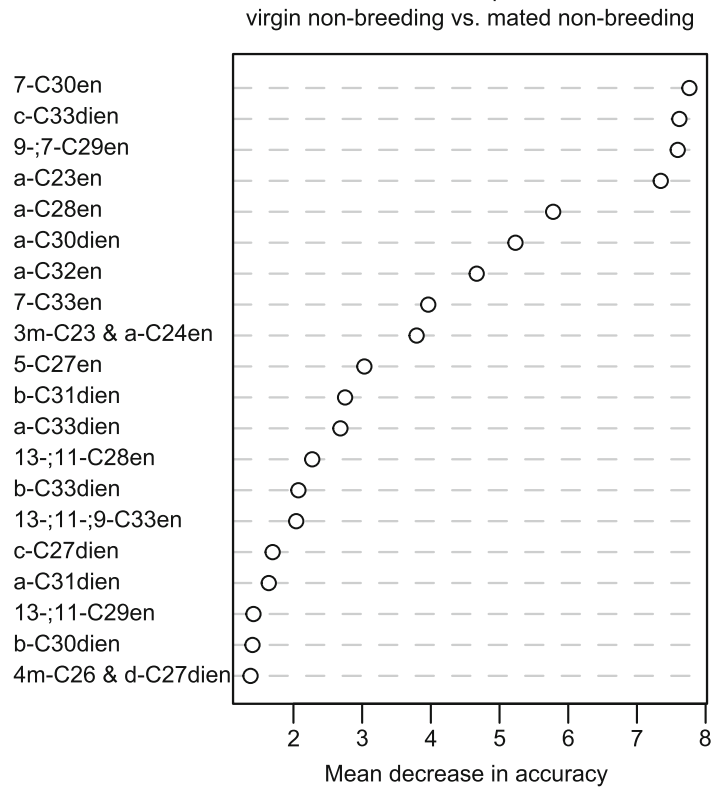

b

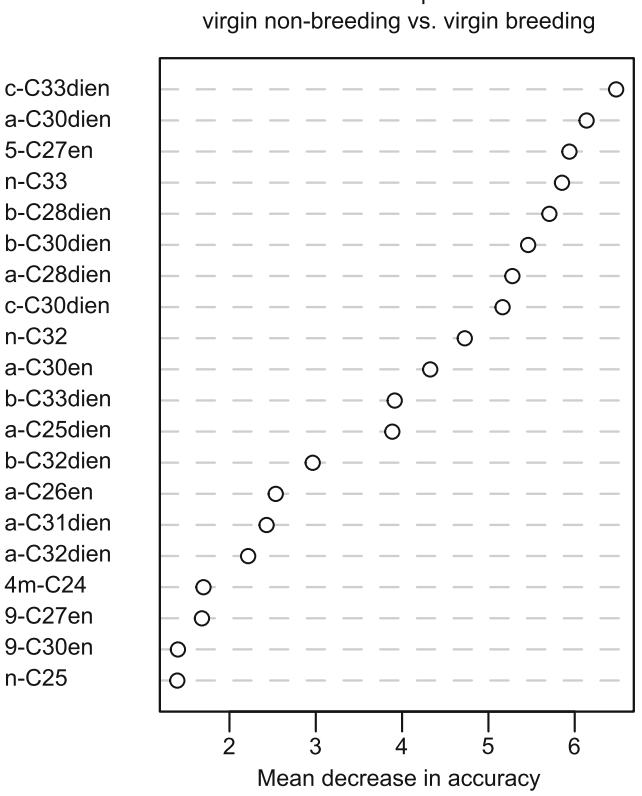

Figure 4. Variable importance plot from a random forest classification of cuticular hydrocarbons used in the identification of group membership of two groups of female bumblebees: a virgin nonbreeding queens versus mated nonbreeding queens, $\mathbf{b}$ virgin nonbreeding queens versus virgin breeding queens. Higher values of mean decrease in accuracy indicate higher importance of the variable for classification.

CHC profiles of virgin nonbreeding queens and foraging workers are the most similar ones among all groups. As in both groups, the individuals are unfertilized and have undeveloped ovaries, the only difference between them is age and caste membership showing that these effects are not major contributors to the separation of the $\mathrm{CHC}$ profile. Therefore, even if we consider an effect of age on the $\mathrm{CHC}$ profiles in $B$. terrestris queens, the effect of breeding status and insemination seems to be more pronounced. Differences in the cuticular profiles between workers and nest-searching queens in B. terrestris have been already shown in a previous study by Sramkova et al. (2008). In their study, however, hydrocarbons and polar compounds have not been analyzed separately. In our study, we analyzed solely cuticular hydrocarbons, and the inclusion of foraging workers allows us to set other influences like ovary development and insemination into perspective.

We conclude that the $\mathrm{CHC}$ profile in $B$. terrestris queens is affected by both ovary development and insemination. The method of anesthetizing and stimulating virgin queens to found a colony gives one the opportunity to investigate the effect of insemination in bumblebees. While we are able to find a strong effect of ovary development on the CHC profile in our study, insemination also seems to have a major effect on the $\mathrm{CHC}$ profile, and therefore, the effect of insemination on potential chemical cues in bumblebees should not be underestimated. The alteration of $\mathrm{CHCs}$ by insemination might also be received by workers as a cue for fertility independent from ovary development. To test whether insemination and depletion of stored sperm in bumblebee queens could have an effect on the switch point and/or the competition point via $\mathrm{CHC}$ alterations, further experiments and bioassays are needed. Further studies could also investigate the effect of the breeding status and insemination on polar compounds of the cuticle of bumblebees. Lastly, stimulating virgin individuals of other social and nonsocial insects and using them for 
similar experiments as shown here might help to improve our understanding of the evolution of chemical cues in insects.

\section{ACKNOWLEDGMENTS}

We are thankful to two anonymous reviewers for their valuable comments on earlier drafts of this paper.

\section{Démêler l'effet de l'insémination et du développement ovarien sur le profile des hydrocarbures cuticulaires chez le bourdon Bombus terrestris (Hymenoptera : Apidae )}

statut de reproduction / cuticule / fertilité / accouplement / reine vierge

Auftrennung des Einflusses von Besamung und O vari e n t w i c k l ung a u d a s kutikulare Kohlenwasserstoffprofil der Erdhummel Bombus terrestris (Hymenoptera : Apidae)

\section{Fortpflanzungsstatus / Kutikulaprofil / Fertilität / Paarung / Jungkönigin}

\section{REFERENCES}

Aitchison, J. (1986) The Statistical Analysis of Compositional Data. Chapman \& Hall, London

Ayasse, M., Jarau, S. (2014) Chemical ecology of bumble bees. Annu. Rev. Entomol. 59 (1), 299-319

Ayasse, M., Maelovits, T., Tengö, J., Taghizadeh, T., Francke, W. (1995) Are there pheromonal dominance signals in the bumblebee Bombus hypnorum L. (Hymenoptera. Apidae)? Apidologie 26(1), $163-180$

Baer, B., Schmid-Hempel, P. (2005) Sperm influences female hibernation success, survival and fitness in the bumble-bee Bombus terrestris. Proc. R. Soc. B. 272 (1560), 319-323

Bagnères, A.G., Wicker-Thomas, C. (2010) Chemical taxonomy with hydrocarbons. In: Blomquist, G.J., Bagnères, A.G. (eds.) Insect hydrocarbons. Biology, biochemistry, and chemical ecology, pp. 121-162. Cambridge University Press, Cambridge

Bloch, G., Hefetz, A., Hartfelder, K. (2000) Ecdysteroid titer, ovary status, and dominance in adult worker and queen bumble bees (Bombus terrestris ). J. Insect Physiol. 46 (6), 1033-1040

Blomquist, G.J., Bagnères, A.G. (2010) Insect Hydrocarbons. Biology, Biochemistry, and Chemical Ecology. Cambridge University Press, Cambridge
Breiman, L. (2001) Random forests. Machine learning $45(1), 5-32$

R Core Team (2014) R: A language and environment for statistical computing. R Foundation for Statistical Computing, Vienna, Austria. URL http://www.R-project.org/

Cutler, D.R., Edwards Jr., T.C., Beard, K.H., Cutler, A., Hess, K.T., Gibson, J., Lawler, J.J. (2007) Random forests for classification in ecology. Ecology 88(11), 2783-2792

Cuvillier-Hot, V., Cobb, M., Malosse, C., Peeters, C. (2001) Sex, age and ovarian activity affect cuticular hydrocarbons in Diacamma ceylonense, a queenless ant. J. Insect Physiol. 47 (4), 485-493

Cuvillier-Hot, V., Gadagkar, R., Peeters, C., Cobb, M. (2002) Regulation of reproduction in a queenless ant: Aggression, pheromones and reduction in conflict. Proc. R. Soc. Lond. B. 269 (1497), 1295-1300

Dietemann, V., Peeters, C., Liebig, J., Thivet, V., Hölldobler, B. (2003) Cuticular hydrocarbons mediate recognition of queens and reproductive workers in the ant Myrmecia gulosa. Proc. Natl. Acad. Sci. USA 100 (18), 10341-10346

Dietemann, V., Liebig, J., Hölldobler, B., Peeters, C. (2005) Changes in the cuticular hydrocarbons of incipient reproductives correlate with triggering of worker policing in the bulldog ant Myrmecia gulosa. Behav. Ecol. Sociobiol. 58(5), 486-496

Duchateau, M.J., Velthuis, H.H.W. (1988) Development and reproductive strategies in Bombus terrestris colonies. Behaviour 107 (3/4), 186-207

Dunkelblum, E., Tan, S.H., Silk, P.J. (1985) Doublebond location in monounsaturated fatty acids by dimethyl disulfide derivatization and mass spectrometry: Application to analysis of fatty acids in pheromone glands of four Lepidoptera. J. Chem. Ecol. 11 (3), 265-277

Everaerts, C., Farine, J.P., Cobb, M., Ferveur, J.F. (2010) Drosophila cuticular hydrocarbons revisited: mating status alters cuticular profiles. PloS one 5(3), e9607

Geva, S., Hartfelder, K., Bloch, G. (2005) Reproductive division of labor, dominance, and ecdysteroid levels in hemolymph and ovary of the bumble bee Bombus terrestris . J. Insect Physiol. 51 (7), 811-823

Gibbs, A.G. (2002) Lipid melting and cuticular permeability: new insights into an old problem. J. Insect. Physiol. 48(4), 391-400

Greeff, M., Schmid-Hempel, P. (2008) Sperm reduces female longevity and increases melanization of the spermatheca in the bumblebee Bombus terrestris L. Insectes Soc. 55(3), 313-319

Harrell, F. E. Jr, and with contributions from many other users (2011) Hmisc: Harrell Miscellaneous. R package version 3.9-0. URL http://CRAN.R-project.org/ package $=$ Hmisc

Heinze, J., Stengl, B., Sledge, M.F. (2002) Worker rank, reproductive status and cuticular hydrocarbon signature in the ant, Pachycondyla cf. inversa. Behav. Ecol. Sociobiol. 52 (1), 59-65 
Howard, R.W., Blomquist, G.J. (1982) Chemical ecology and biochemistry of insect hydrocarbons. Annu. Rev. Entomol. 27(1), 149-172

Howard, R.W., Blomquist, G.J. (2005) Ecological, behavioural, and biochemical aspects of insect hydrocarbons. Annu. Rev. Entomol. 50, 371-393

Keller, L., Nonacs, P. (1993) The role of queen pheromones in social insects - Queen control or queen Signal. Animal Behav. 45(4), 787-794

Liaw, A., Wiener, M. (2002) Classification and Regression by randomForest. R News 2 (3), 18-22

Liebig, J. (2010) Hydrocarbon profiles indicate fertility and dominance status in ant, bee, and wasp colonies. In: Blomquist, G.J., Bagnères, A.G. (eds.) Insect hydrocarbons. Biology, biochemistry, and chemical ecology, pp. 254-281. Cambridge University Press, Cambridge

Liebig, J., Peeters, C., Oldham, N.J., Markstädter, C., Hölldobler, B. (2000) Are variations in cuticular hydrocarbons of queens and workers a reliable signal of fertility in the ant Harpegnathos saltator? Proc. Natl. Acad. Sci. USA 97 (8), 4124-4131

Lockey, K.H. (1988) Lipids of the insect cuticle: origin, composition and function. Comp. Biochem. Phys. B. 89 (4), 595-645

Lommelen, E., Johnson, C.A., Drijfhout, F.P., Billen, J., Wenseleers, T., Gobin, B. (2006) Cuticular hydrocarbons provide reliable cues of fertility in the ant Gnamptogenys striatula. J. Chem. Ecol. 32 (9), 2023-2034

Monnin, T., Malosse, C., Peeters, C. (1998) Solid-phase microextraction and cuticular hydrocarbon differences related to reproductive activity in queenless ant Dinoponera quadriceps. J. Chem. Ecol. 24(3), 473490

Mpuru, S., Blomquist, G.J., Schal, C., Roux, M., Kuenzli, M., Dusticier, G., Clément, J.-L., Bagnères, A.G. (2001) Effect of age and sex on the production of internal and external hydrocarbons and pheromones in the housefly, Musca domestica. Insect Biochem. Mol. Biol. 31 (2), 139-155

Niño, E.L., Malka, O., Hefetz, A., Tarpy, D.R., Grozinger, C.M. (2013) Chemical profiles of two pheromone glands are differentially regulated by distinct mating factors in honey bee queens (Apis mellifera L.). PloS one 8 (11), e 78637

Oksanen, J., Blanchet, F. G., Kindt, R., Legendre, P., Minchin, P. R., O'Hara, R. B., Simpson, G. L., Solymos, P., Stevens, M. H. H., Wagner, H. (2011) vegan: Community Ecology Package. R package version 2.0-2. URL http:/CRAN.Rproject.org/package=vegan

Panek, L.M., Gamboa, G.J., Espelie, K.E. (2001) The Effect of a Wasp's Age on Its Cuticular Hydrocarbon Profile and Its Tolerance by Nestmate and Non-
Nestmate Conspecifics (Polistes fuscatus, Hymenoptera: Vespidae). Ethology 107 (1), 55-63

Peeters, C., Monnin, T., Malosse, C. (1999) Cuticular hydrocarbons correlated with reproductive status in a queenless ant. Proc. R. Soc. Lond. B. 266 (1426), 1323-1327

Richard, F.J., Schal, C., Tarpay, D.R., Grozinger, C.M. (2011) Effects of instrumental insemination and insemination auantity on Dufour's gland chemical profiles and Vitellogenin expression in honey bee queens (Apis mellifera ). J. Chem. Ecol. 37 (9), 1027-1036

Robinson, G.E. (1992) Regulation of division of labor in insect societies. Annu. Rev. Entomol. 37 (1), 637-665

Röseler, P.F., van Honk, C.G.J. (1990) Castes and reproduction in bumblebees. In: Engels, W. (ed.) Social insects, an evolutionary approach to castes and reproduction, pp. 147-166. Springer, Berlin

Sledge, M.F., Boscaro, F., Turillazzi, S. (2001) Cuticular hydrocarbons and reproductive status in the social wasp Polistes dominulus. Behav. Ecol. Sociobiol. 49 (5), 401-409

Sledge, M.F., Trinca, I., Massolo, A., Boscaro, F., Turillazzi, S. (2004) Variation in cuticular hydrocarbon signatures, hormonal correlates and establishment of reproductive dominance in a polistine wasp. J. Insect Physiol. 50(1), 73-83

Smith, A.A., Hölldobler, B., Liebig, J. (2008) Hydrocarbon signals explain the pattern of worker and egg policing in the ant Aphaenogaster cockerelli. J. Chem. Ecol. 34 (10), 1275-1282

Smith, A.A., Hölldobler, B., Liebig, J. (2009) Cuticular hydrocarbons reliably identify cheaters and allow enforcement of altruism in a social insect. Curr. Biol. 19 (1), 78-81

Smith, A.A., Millar, J.G., Suarez, A.V. (2015) A social insect fertility signal is dependent on chemical context. Biol. Lett. 11 (1), 20140947

Sramkova, A., Schulz, C., Twele, R., Francke, W., Ayasse, M. (2008) Fertility signals in the bumblebee Bombus terrestris (Hymenoptera: Apidae). Naturwissenschaften 95 (6), 515-522

St. Leger, R.J. (1995) The role of cuticle-degrading proteases in fungal pathogenesis of insects. Can. J. Botany 73 (S1), 1119-1125

van Zweden, J.S., d'Ettorre, P. (2010) Nestmate recognition in social insects and the role of hydrocarbons. In: Blomquist, G.J., Bagnères, A.G. (eds.) Insect hydrocarbons. Biology, biochemistry, and chemical ecology, pp. 222-243. Cambridge University Press, Cambridge

Venables, W.N., Ripley, B.D. (2002) Modern Applied Statistics with S, 4th edn. Springer, New York

Wilson, E.O. (1963) The social biology of ants. Annu. Rev. Entomol. 8(1), 345-368 